\title{
Self-Energies for Interacting Fields with a Compactified Spatial Dimension
}

\author{
F. A. Barone* \\ ICE - Universidade Federal de Itajubá, Av. BPS 1303, Caixa Postal 50, 37500-903, Itajubá, MG, Brazil. \\ M. Hott ${ }^{\dagger}$ \\ Departamento de Física e Química, UNESP-Campus de Guaratinguetá, 12500-000, Guaratinguetá, SP, Brazil.
}

Received on 4 November, 2007

\begin{abstract}
We study the self-energy of a model with a tri-linear coupling among spinless fields on a manifold with one spatial dimension compactified. The model is considered to be composed by a quantized scalar field interacting with a classical scalar one. In order to improve the compactification we take the quantum field satisfying quasi periodic boundary conditions, which interpolates continuously the periodic and anti-periodic conditions, whilst the background field satisfies periodic boundary conditions. All the calculations are performed in Euclidean coordinates.
\end{abstract}

Keywords: Quantum fields; Compactified dimensions

\section{INTRODUCTION}

Since the seminal papers of T. Kaluza [1] and O. Klein [2], there have been many studies of models with extra dimensions in the context of strings theory and development of works investigating models with compactified spatial dimensions in the context of Quantum Field Theory. In this scenario we can cite, for instance, studies about the proton stability in six dimensions [3], grand unified theories in extra dimensions [4], the descriptions of the Higgs particle from models with extra dimensions [5], a justification of the Electroweak symmetry breaking with extra spatial dimensions compactified and without the introductions of the Higgs field [6, 7], the development of spontaneous broken symmetry of spaces with compactified dimensions [8], the role of the Casimir effect in the radius stabilization of extra dimensions [9], the gauge invariance of massive modes of quantum fields in theories with extra dimensions compactified [10], thermal effects of fields with spatial dimensions compactified [11], the existence of an unbroken subgroup of the six-dimensional Lorentz symmetry for models with chiral compactification [12]. Other examples are the studies of the influence of boundary conditions in the cosmological scenario [13], in the corrections to the masses of fields $[14,16]$, in symmetry breaks $[17,18]$ and in the renormalization of quantum field theories in non-simply connected space-time [19].

In the context of Quantum Field Theory it is relevant to investigate if the existence of extra compactified dimensions could produce some different characteristics on systems (which could be controlled in the laboratory) in comparison to the situation where extra dimensions do not exist. A good scenario which can be of some inspiration for such kinds of phenomena is the Quantum Field Theory at Finite Temperature (QFTFT), once in the imaginary time formalism we have one of the dimensions, the time dimension, compactified. In

\footnotetext{
*e-mail: fbarone@unifei.edu.br

†e-mail: hott@feg.unesp.br
}

this sense, models of QFTFT can exhibit narrow similarities with corresponding ones in Quantum Field Theory in 3+1 dimensions at zero temperature but with one of the spatial dimension compactified.

It is well known that in quantum field theory at finite temperature (QFTFT), some Feynman diagrams of the theory can be non-analytical $[20,21]$, in the sense that, the zero limit of the external momentum is not well defined. In other words, the limit $\Pi\left(k_{0}=0, \mathbf{k} \rightarrow 0\right)$ does not commute with the limit $\Pi\left(\mathbf{k}=0, k_{0} \rightarrow 0\right)$, where $\Pi\left(k_{0}, \mathbf{k}\right)$ designates a Feynman amplitude and $k_{0}(\mathbf{k})$ stands for the time (space) component of external momentum. Such non-analyticity is due to a branch cut along the real axis of the complex plane of the timecomponent of the external momentum, with branch point at the origin, if the internal propagators of the loop carries the same mass [22]. The branch point is the threshold above which the amplitude for the decay of real particles into "thermal particles", and vice versa, is different from zero. The nonanalyticity can be seen in any one of the formalisms used to treat QFTFT, but the doubling of degrees of freedom is manifested naturally in the real-time formalisms. The real-time formalisms are also appropriate settings to analyze effective actions at finite temperature, since the derivatives of any field, in terms of which one express the effective Lagrangian, are assumed to be continuous and there is no need to resort to analytic continuation from discrete to continuous momenta. Unfortunately, even the derivative expansion of the effective Lagrangian is plagued with the non-analyticity that renders it essentially non-local [23], unless the external field is static. The imaginary-time formalism in quantum field theory at finite temperature is also thoroughly used to analyze effective potentials and thermal corrections to the masses of particles.

A natural question that can be raised is: in analogy with QFTFT, how the non-commutativity is manifested and which kind of phenomena it can produce when one considers quantum field theory at zero temperature but with one spatial dimension compactified. This could be an interesting subject in the study of systems defined on manifolds with one spatial dimension compactified, like nanoubes, and also in KaluzaKlein theories. 
Due these considerations, in this work we analyze the influence of boundary conditions on the quantum correction to the mass of a real classical scalar field interacting with a quantized complex scalar field [24]. The quantum field satisfies quasi-periodic boundary conditions, which interpolates continuously the periodic and anti-periodic cases in one of the spatial dimensions. The classical field is taken to be satisfying periodic boundary condition along the same spatial direction. To keep an analogy with QFTFT the calculations are performed in Euclidean time in $3+1$ dimensions. We analyze the behavior of the self-energy diagram as a function of the modulus and direction of the external momentum, and point out the possible origin for the non-commutativity. We show that the correction to the masses for directions of propagation perpendicular and parallel to the compactified axis can be the same depending on how the limit of the external momentum going to zero is taken. In other words, it is shown that the limits $\Pi\left(k_{\|}=0, k_{\perp} \rightarrow 0\right)$ and $\Pi\left(k_{\perp}=0, k_{\|} \rightarrow 0\right)$, where $k_{\|}\left(k_{\perp}\right)$ is the component of the external momentum parallel (transverse) to the compactified dimension [27], commute. The first (second) of the limits is considered when the field propagates in the direction transverse (parallel) to the compactified axis. We also show that this commutativity does not occur for an otherwise arbitrary direction of propagation.

We consider this specific model because it was studied previously in the context QFTFT in the analysis of noncommutativity and effective action [23]. The graphics for the self-energy in this model is simpler than the photon selfenergy in the QED, for instance, which exhibits a tensor structure. Furthermore, the chosen boundary conditions interpolate continuously the periodic and anti-periodic cases and its role in the non-commutativity of self-energy graphics were not studied in the literature previously, although similar boundary conditions in QFTFT in $0+1$ dimensions were studied in the context of interpolating statistics $[25,26]$.

\section{THE SELF-ENERGY}

We shall consider a model which is described, in Euclidean coordinates, by the Lagrangian density

$$
\mathcal{L}=\left(\partial_{\mu} \phi\right)\left(\partial_{\mu} \phi^{*}\right)+m^{2}|\phi|^{2}+\frac{1}{2}\left(\partial_{\mu} B\right)^{2}+\frac{1}{2} M^{2} B^{2}+g B|\phi|^{2},
$$

with $\phi \equiv \phi(x)$ being a quantized complex scalar field, $B \equiv B(x)$ a real classical scalar field (not quantized) and $g$ a coupling constant. We also consider that the $B(x)$ classical field satisfies periodic boundary condition in the third spatial coordinate $x_{3}$

$$
B\left(x_{0}, x_{1}, x_{2}, x_{3}\right)=B\left(x_{0}, x_{1}, x_{2}, x_{3}+a\right),
$$

and the $\phi(x)$ satisfies the so called quasi-periodic condition

$$
\phi\left(x_{0}, x_{1}, x_{2}, x_{3}\right)=e^{i 2 \pi \theta} \phi\left(x_{0}, x_{1}, x_{2}, x_{3}+a\right) .
$$

In the condition above $\theta$ is a parameter whose value is in the interval $0 \leq \theta<1$, and $a$ is the compactifying length. Note that for $\theta=0$ and $\theta=1 / 2$ we recover the periodic and antiperiodic boundary conditions, respectively. Similar boundary conditions have been imposed in the imaginary time to present systems that obey an interpolating statistics [25] and also to analyze the equation of state of a gas of particles obeying an interpolating statistics [26].

In the appendix we use perturbation theory with fields satisfying finite boundary conditions, in order to write the effective Lagrangian density for the classical field $B(x)$, up to second order in the coupling constant $g$, as

$$
\mathcal{L}_{e f f}=\frac{1}{2}\left(\partial_{\mu} B\right)^{2}+\frac{1}{2} M^{2} B^{2}+\frac{1}{2} B(x)(\Pi(\partial)) B(x),
$$

where the self-energy $\Pi(\partial)$ is given by the differential operator

$$
\Pi(\partial)=g^{2} \int \frac{d^{3} \mathbf{p}_{\perp}}{(2 \pi)^{3}} \frac{1}{a} \sum_{\ell=-\infty}^{\infty}\left\{\frac{1}{\mathbf{p}_{\perp}^{2}+\left[\frac{2 \pi}{a}(\ell+\theta)\right]^{2}+m^{2}} \frac{1}{\left(\mathbf{p}_{\perp}-i \partial_{\perp}\right)^{2}+\left[\frac{2 \pi}{a}(\ell+\theta)-i \partial_{3}\right]^{2}+m^{2}}\right\}
$$

with $\partial_{\perp}=\left(\partial_{0}, \partial_{1}, \partial_{2}\right)$ the derivative with respect to the coordinates transverse to the compactified axis, $\partial_{3}$ the derivative with respect to the compactified coordinate $x_{3}$ and $\mathbf{p}_{\perp}=\left(p_{0}, p_{1}, p_{2}\right)$. The above expression is written in terms of derivatives of the external field $B(x)$ and the derivatives in the equation (4) act to the right. The derivative expansion, which constitutes in a Taylor series of $\Pi\left(a, \theta, m, \partial_{3}\right)$ in powers of $\partial / m$, is useful to obtain a local effective Lagrangian for that field. Later we are going to see that, due to the non-analyticity of the self-energy in the origin of the external momenta, a derivative expansion of the effective lagrangian is not well-defined.

In the Appendix we show that in the momenta space the effective action integral for $B(x)$ becomes

$$
\begin{aligned}
S_{e f f} & =-\int d^{4} x \mathcal{L}_{e f f}= \\
& =-\frac{1}{2} \int \frac{d^{3} \mathbf{k}_{\perp}}{(2 \pi)^{3}} \sum_{n=-\infty}^{\infty} \tilde{B}_{-n}\left(-\mathbf{k}_{\perp}\right)\left[\mathbf{k}_{\perp}^{2}+\left(\frac{2 n \pi}{a}\right)^{2}+M^{2}+\tilde{\Pi}\left(\mathbf{k}_{\perp}, n\right)\right] \tilde{B}_{n}\left(\mathbf{k}_{\perp}\right),
\end{aligned}
$$

where $\mathbf{k}_{\perp}=\left(k_{0}, k_{1}, k_{2}\right)$ is the component of the momentum of the field $B(x)$ in the direction transverse to the $x_{3}$ axis, $\tilde{B}_{n}\left(\mathbf{k}_{\perp}\right)$ is 
the Fourier transform of the field $B(x)$ and

$$
\tilde{\Pi}\left(\mathbf{k}_{\perp}, n\right)=g^{2} \int \frac{d^{3} \mathbf{p}_{\perp}}{(2 \pi)^{3}} \frac{1}{a} \sum_{\ell=-\infty}^{\infty}\left\{\frac{1}{\mathbf{p}_{\perp}^{2}+\left[\frac{2 \pi}{a}(\ell+\theta)\right]^{2}+m^{2}} \frac{1}{\left(\mathbf{p}_{\perp}+\mathbf{k}_{\perp}\right)^{2}+\left[\frac{2 \pi}{a}(\ell+\theta)+\frac{2 \pi n}{a}\right]^{2}+m^{2}}\right\}
$$

is the self-energy in the momenta space. The set of vectors $\left(\mathbf{p}_{\perp}, p_{3}=\frac{2 \pi}{a}(\ell+\theta)\right)$ and $\left(\mathbf{k}_{\perp}, 2 \pi n / a\right)$ are the momenta of the fields $\phi(x)$ and $B(x)$ respectively.

The summation which appears in (7) is over integer values of $l$ and can be calculated using the expression

$$
\sum_{\ell=-\infty}^{\infty} f(\ell)=-\pi \sum_{\substack{\text { poles } \\ f(z)}} \operatorname{Res}[f(z) \cot (\pi z)]
$$

which is valid for any function $f(z)$ that does not have poles at $z=0, \pm 1, \pm 2, \ldots$ and has the $\operatorname{limit}_{\lim _{|z| \rightarrow \infty}} f(z)=0$ [21] In expression (8) the summation on the right hand side is over the poles of $f(z)$, with $z$ a complex variable. By using the fact that

$$
\operatorname{coth}(z)=1+2 n_{B}(2 z)
$$

where $n_{B}(z)$ is the Bose-Einstein distribution function (B-E factor),

$$
n_{B}(z)=\frac{1}{\exp (z)-1}
$$

the equation (7) can be split into two contributions

$$
\tilde{\Pi}\left(\mathbf{k}_{\perp}, n\right)=\tilde{\Pi}\left(k^{2}\right)+\tilde{\Pi}\left(a, \theta, \mathbf{k}_{\perp}, k_{3}\right)
$$

where the first term is the sole contribution to the self-energy without boundary conditions once it depends on neither the length $a$ nor the interpolating parameter $\theta$; the second one is the contribution due to the boundary conditions. From now on we concentrate on this second part of the self-energy which is written as

$$
\begin{gathered}
\tilde{\Pi}\left(a, \theta, \mathbf{k}_{\perp}, k_{3}\right)=\frac{1}{2} \int \frac{d^{3} \mathbf{p}_{\perp}}{(2 \pi)^{3}}\left[\frac{1}{\omega_{\mathbf{p}_{\perp}}} \frac{1}{k^{2}+2\left(\mathbf{k}_{\perp} \cdot \mathbf{p}_{\perp}+i k_{3} \omega_{\mathbf{p}_{\perp}}\right)} n_{B}\left(a \omega_{\mathbf{p}_{\perp}}+2 \pi i \theta\right)+\right. \\
\left.+\frac{1}{\omega_{\mathbf{p}_{\perp}+\mathbf{k}_{\perp}}} \frac{1}{k^{2}-2\left(\mathbf{k}_{\perp} \cdot \mathbf{p}_{\perp}+i k_{3} \omega_{\left.\mathbf{p}_{\perp}+\mathbf{k}_{\perp}\right)}\right.} n_{B}\left(a \omega_{\mathbf{p}_{\perp}+\mathbf{k}_{\perp}}+2 \pi i \theta+i a k_{3}\right)\right]+C . C .
\end{gathered}
$$

with

$$
k^{2}=k_{3}^{2}+\mathbf{k}_{\perp}^{2}, \quad k_{3}=\frac{2 \pi}{a} n, \quad, \quad \omega_{\mathbf{p}_{\perp}}=\sqrt{\mathbf{p}_{\perp}^{2}+m^{2}} \quad \text { and } \quad \omega_{\mathbf{p}_{\perp}+\mathbf{k}_{\perp}}=\sqrt{\mathbf{p}_{\perp}^{2}+m^{2}} .
$$

The C.C. designates the complex conjugate of its preceding terms.

In the equation (12) we make the change of variables $\mathbf{p}_{\perp} \rightarrow-\left(\mathbf{p}_{\perp}+\mathbf{k}_{\perp}\right)$ in the integrals which contains $k_{3}$ in the argument of the B-E factor $n_{B}$, and integrate in the angular variables to obtain

$$
\begin{aligned}
\tilde{\Pi}\left(a, \theta, \mathbf{k}_{\perp}, k_{3}\right) & =\frac{1}{(2 \pi)^{2}} \frac{1}{4 k_{\perp}} \int_{0}^{\infty} d p_{\perp} \frac{p_{\perp}}{\omega_{\mathbf{p}_{\perp}}}\left[\ln \left(\frac{k^{2}+2\left(k_{\perp} p_{\perp}+i k_{3} \omega_{\mathbf{p}_{\perp}}\right)}{k^{2}-2\left(k_{\perp} p_{\perp}-i k_{3} \omega_{p_{\perp}}\right)}\right) n_{B}\left(a \omega_{\mathbf{p}_{\perp}}+2 \pi i \theta\right)+\right. \\
& \left.+\ln \left(\frac{k^{2}+2\left(k_{\perp} p_{\perp}-i k_{3} \omega_{\mathbf{p}_{\perp}}\right)}{k^{2}-2\left(k_{\perp} p_{\perp}+i k_{3} \omega_{\mathbf{p}_{\perp}}\right)}\right) n_{B}\left(a \omega_{\mathbf{p}_{\perp}}+2 \pi i \theta+i a k_{3}\right)\right]+ \text { C.C. }
\end{aligned}
$$

By rotating back to Minkowskian space-time and expanding (14) in powers of the external momenta, one can verify from the expression for the effective action, equation (6), that the zero order term in the external momenta gives the zero order correction, in powers of the external momenta, to the mass of the $B(x)$ field, up to second order in the coupling constant $g$. One can also see that the components of the external momenta, $k_{3}$ and $\mathbf{k}_{\perp}$, are not in the same foot in (14) and the zero-momentum limit depends on the path in the momenta space along which it is taken. We say that based on similar results in QFTFT where a similar picture is present and two masses come out, the plasmon mass due to time oscillations and the Debye mass related to the screening of the external field. Since here the boundary conditions are imposed on one 
of the space coordinates, we associate the zero-momentum limit $\tilde{\Pi}\left(a, \theta, k_{3}=0, \mathbf{k}_{\perp} \rightarrow 0\right)$ with the correction to the mass when the field $B(x)$ propagates in the direction transverse to the $x_{3}$, whilst the limit $\tilde{\Pi}\left(a, \theta, \mathbf{k}_{\perp} \rightarrow 0, k_{3} \rightarrow 0\right)$ is the correction to the mass related to the propagation of the field parallel to the compactified dimension. The first of the limits corresponds to set $k_{3}=0$ first and then to take the limit $\mathbf{k}_{\perp} \rightarrow 0$. On the other hand, the second limit corresponds to take the limit $\mathbf{k}_{\perp} \rightarrow 0$ first and only after the contribution of $n=0$ is evaluated. It worths to mention that the time dimension plays no role in this scenario; as a matter of fact we could suppose the real field to be a static one and, in such a case, we might speak of different Debye screening lengths that are given by the inverse of the "masses", depending on which direction we approach the origin of the momentum space. In the next section the propagation directions perpendicular and parallel to the $x_{3}$ are analyzed in some detail.

\section{THE PARALLEL AND PERPENDICULAR PROPAGATIONS}

In this section we calculate the quantum corrections to the mass of the $B(x)$ field for propagations parallel and perpendicular to the $x_{3}$ axis. For this task, it is convenient to define the dimensionless variables

$$
u=\frac{p_{\perp}}{m}, v=\frac{k_{\perp}}{m}, z=\frac{k_{3}}{m}, s=m a
$$

in order to write equation (14) in the form

$$
\tilde{\Pi}\left(a, \theta, \mathbf{k}_{\perp}, k_{3}\right)=g^{2} \frac{1}{(2 \pi)^{2}} \frac{1}{4} \int_{0}^{\infty} d u \frac{u}{\sqrt{1+u^{2}}} I(z, v, u, s, \theta)
$$

where we have defined

$$
\begin{array}{r}
I(z, v, u, s, \theta)=\frac{1}{v}\left[\ln \left(\frac{v^{2}+z^{2}+2\left(v u+i z \sqrt{1+u^{2}}\right)}{v^{2}+z^{2}-2\left(v u-i z \sqrt{1+u^{2}}\right)}\right)\right. \\
\left.\times\left(n_{B}\left(s \sqrt{1+u^{2}}+2 \pi i \theta\right)+n_{B}\left(s \sqrt{1+u^{2}}-2 \pi i \theta-i s z\right)\right)\right]+C . C .
\end{array}
$$

Notice that the variable $z$ defined in (15) is discrete once $k^{3}$, defined in (13), is a discrete variable.

Let us, first, study the correction to the mass when the propagation is perpendicular to the $x_{3}$ axis. In this case the external momentum is given by $k_{\mu}=\left(\mathbf{k}_{\perp}, 0\right)$, that is, $k_{3}=0$. This amounts to $z=0$ in (17), what leads to

$$
I(z=0, v, u, s, \theta)=\frac{1}{v}\left[\ln \left(\frac{(v+2 u)^{2}}{(v-2 u)^{2}}\right)\left(n_{B}\left(s \sqrt{1+u^{2}}+2 \pi i \theta\right)+n_{B}\left(s \sqrt{1+u^{2}}-2 \pi i \theta\right)\right)\right] .
$$

By substituting (18) into (16) we have the self-energy for a propagation perpendicular to the $x_{3}$ axis, whose zero-momentum limit, $\mathbf{k}_{\perp} \rightarrow 0$, gives the zero-order correction, in powers of $v^{2}=k_{\perp}^{2} / m^{2}$, to the mass for the $B(x)$ field. This limit is equivalent to take $v \rightarrow 0$, that is

$$
\lim _{v \rightarrow 0} I(z=0, v, u, s, \theta)=\frac{2}{u}\left(n_{B}\left(s \sqrt{1+u^{2}}+2 \pi i \theta\right)+n_{B}\left(s \sqrt{1+u^{2}}-2 \pi i \theta\right)\right)
$$

By using (16), (19) and the definitions (15) we have

$$
\lim _{k_{\perp} \rightarrow 0} \tilde{\Pi}^{\prime}\left(a, \theta, \mathbf{k}_{\perp}=0, k_{3}\right)=g^{2} \frac{1}{(2 \pi)^{2}} \frac{1}{2} \int_{0}^{\infty} d u \frac{1}{\sqrt{1+u^{2}}} n_{B}\left(m a \sqrt{1+u^{2}}+2 \pi i \theta\right)+C . C .
$$

We would have obtained the same result if we had set the external momenta equal zero from the very beginning, that is, if we had assumed $\mathbf{k}_{\perp}=0$ and $n=0$ in the expression (7), but this procedure is not reliable since the variables $z$ and $v$ are not treated in the same foot and the integrand of (16) is not analytic in the origin of the $(z, v)$ plane. This can be seen by taking the limit $v \rightarrow 0$ and then set $z=0$.

Now, let us consider the correction to the mass when the propagation is parallel to the $x_{3}$ axis. In this situation the external momentum is given by $k_{\mu}=\left(0, k_{3}\right)$, that is, $\mathbf{k}_{\perp}=0$, or equivalently $v=0$. So, by taking $v=0$ in the expression (17) we obtain

$$
I(z, v=0, u, s, \theta)=\frac{4 u}{z\left(z+2 i \sqrt{1+u^{2}}\right)}\left(n_{B}\left(s \sqrt{1+u^{2}}+2 \pi i \theta\right)+n_{B}\left(s \sqrt{1+u^{2}}-2 \pi i \theta-i s z\right)\right)+C . C .
$$

The above expression evaluated at $z=0$ furnishes us the zero-order correction, in powers of $z^{2}=k_{3}{ }^{2} / \mathrm{m}^{2}$, to the mass of the external field when the propagation is parallel to the 
compactified axis. One can see that the above expression is not well defined for $n=0$, so we need a different prescription for $I(z, v=0, u, s, \theta)$ which can obtained by constructing a function of the continuous variable $z$ defined in such a way that, when $z=2 \pi /(m a) n$, it coincides with the values given by the right hand side of (21). In addition, this new function must have a well defined limit when $z=0$.

In order to do that it is important to notice that the last term in the argument of the second B-E factor of the expression (21) (the same factor is also present in the C.C. terms) can be rewritten as $i s z=2 \pi n$. Then, from (10), one can conclude that they contribute a factor $\exp ( \pm i 2 \pi n)=1$ for any $n$ integer and as such, does not influence the result of $I(z, v=0, u, s, \theta) \|_{z=0}$. As a matter of fact this argument is correct only for $n \neq 0$, but not for $n=0$, since each term of $I(z, v=0, u, s, \theta)$ exhibits divergences in $n=0$. In other words, once expression (21) is well defined for $n \neq 0$ one can put $\exp ( \pm i 2 \pi n)=1$, for $n=0$ this expression is ill defined, so we need another prescription for $I(z, v=0, u, s, \theta)$ in this case.

The presence of the the term $\exp ( \pm i 2 \pi n)$ in equation (21) plays a central role in the final results, as shall be exposed. If one takes $\exp ( \pm i 2 \pi n)=1$ in the expression (21) for any value of $n$ (including $n=0$ ) one obtains

$$
\begin{aligned}
I^{\prime}(z, v=0, u, s, \theta) & =\frac{4 u}{z\left(z+2 i \sqrt{1+u^{2}}\right)}\left(n_{B}\left(s \sqrt{1+u^{2}}+2 \pi i \theta\right)+n_{B}\left(s \sqrt{1+u^{2}}-2 \pi i \theta\right)\right)+C . C .= \\
& =\frac{8 u}{z^{2}+4\left(1+u^{2}\right)}\left(n_{B}\left(s \sqrt{1+u^{2}}+2 \pi i \theta\right)+n_{B}\left(s \sqrt{1+u^{2}}-2 \pi i \theta\right)\right)
\end{aligned}
$$

which has a well defined value for $z=0$, namely

$$
\left.I^{\prime}(z, v=0, u, s, \theta)\right|_{z=0}=\frac{2 u}{\left(1+u^{2}\right)}\left(n_{B}\left(s \sqrt{1+u^{2}}+2 \pi i \theta\right)+n_{B}\left(s \sqrt{1+u^{2}}-2 \pi i \theta\right)\right) .
$$

By substituting this result in the expression (16), one can deduce that the screening mass for the field propagating along the $x_{3}$ direction is given by

$$
\left.\tilde{\Pi}^{\prime}\left(a, \theta, m, \mathbf{k}_{\perp}, k_{3}\right)\right|_{k_{3}=0}=g^{2} \frac{1}{(2 \pi)^{2}} \frac{1}{2} \int_{0}^{\infty} d u \frac{u^{2}}{1+u^{2}} n_{B}\left(m a \sqrt{1+u^{2}}+2 \pi i \theta\right)+C . C .
$$

It is worth mentioning that the prime in equations (23) and (24) means that these quantities where obtained by taking $\exp ( \pm i 2 \pi n)=1$ in equation (21) and setting $z=0$ after that.

One can see that the corrections to the mass given by expressions (20) and (24) do not coincide with each other. We say that the limit $k_{3}=0, \mathbf{k}_{\perp} \rightarrow 0$ does not commute with the limit $\mathbf{k}_{\perp} \rightarrow 0, k_{3}=0$.

Note that in obtaining the result (24) we are assuming that in the expression (22) $z$ is a continuous variable and the expression

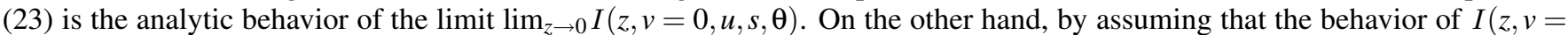
$0, u, s, \theta)$ for small values of $k_{3}\left(k_{3}<<2 \pi / a\right)$ must be analytically continued by taking into account the contribution of $\exp ( \pm i s z)$, we have that

$$
\begin{aligned}
\lim _{z \rightarrow 0} I(z, v=0, u, s, \theta) & =\frac{2 u}{\sqrt{1+u^{2}}} \times\left[\frac{1}{\sqrt{1+u^{2}}} n_{B}\left(s \sqrt{1+u^{2}}+2 \pi i \theta\right)\right. \\
& \left.+s \exp \left(s \sqrt{1+u^{2}}+2 i \pi \theta\right)\left[n_{B}\left(s \sqrt{1+u^{2}}+2 \pi i \theta\right)\right]^{2}\right]+ \text { C.C. }
\end{aligned}
$$

which differs from the result in (23) by the term involving the square of the B-E factor.

By substituting (25) into (16) we have

$$
\begin{array}{r}
\lim _{k_{3} \rightarrow 0} \tilde{\Pi}\left(a, \theta, m, \mathbf{k}_{\perp}=0, k_{3}\right)=g^{2} \frac{1}{(2 \pi)^{2}} \frac{1}{4} \int_{-\infty}^{\infty} d u u\left[\frac{u}{\left(1+u^{2}\right)^{3 / 2}} n_{B}\left(s \sqrt{1+u^{2}}+2 \pi i \theta\right)\right. \\
\left.+\frac{s u}{1+u^{2}} \exp \left(s \sqrt{1+u^{2}}+2 i \pi \theta\right)\left[n_{B}\left(s \sqrt{1+u^{2}}+2 \pi i \theta\right)\right]^{2}\right]+ \text { C.C. }
\end{array}
$$

which can also be written in the form

$$
\lim _{k_{3} \rightarrow 0} \tilde{\Pi}\left(a, \theta, m, \mathbf{k}_{\perp}=0, k_{3}\right)=-g^{2} \frac{1}{(2 \pi)^{2}} \frac{1}{4} \int_{-\infty}^{\infty} d u u \frac{d}{d u}\left[\frac{1}{\sqrt{1+u^{2}}} n_{B}\left(s \sqrt{1+u^{2}}+2 \pi i \theta\right)\right]+C . C .
$$


By performing an integration by parts and using (15) and (20), it can be shown that

$$
\begin{aligned}
\lim _{k_{3} \rightarrow 0} \tilde{\Pi}\left(a, \theta, m, \mathbf{k}_{\perp}=0, k_{3}\right) & =g^{2} \frac{1}{(2 \pi)^{2}} \frac{1}{2} \int_{0}^{\infty} d u \frac{1}{\sqrt{1+u^{2}}} n_{B}\left(m a \sqrt{1+u^{2}}+2 \pi i \theta\right)+C . C . \\
& =\lim _{k_{\perp} \rightarrow 0} \tilde{\Pi}\left(a, \theta, m, \mathbf{k}_{\perp}, k_{3}=0\right) .
\end{aligned}
$$

That is, the correction to the mass generated when the propagation is parallel to the $x_{3}$ axis is the same as the one generated in a propagation perpendicular to the $x_{3}$ axis.

At this point we would like to mention that in the reference [17] a gauge invariant mass term for real photons propagating in the direction perpendicular to the compactified axis is also found due to vacuum polarization. By working with Feynman parametrization to calculate the effects of twisted and untwisted spinors L. Ford found an expression for the vacuum polarization tensor that is analytic in the origin of momenta space and, as a result, the photon mass generated dynamically is unique. In this section we have shown the origin of the nonanalyticity on the self-energy but have also shown how to circumvent this problem by considering an analytic continuation for the self-energy for small values of the external momenta and, as a consequence, we have also found a unique result for the correction to the mass of the real scalar field.

We notice that a derivative expansion of the effective action is still put in jeopardy, because the next coefficients in the Taylor series exhibits a more severe non-analyticity. Once each of those coefficients is obtained from derivatives of the self-energy evaluated at zero-momentum, and each derivative results in one extra power of $k$ in the numerator, the zeromomentum limit of these higher power of momenta in the numerator dominate over the zero-momentum limit of the B-E factors.

\section{SELF-ENERGY FOR AN ARBITRARY PROPAGATION DIRECTION}

In this section we study the behavior of the zero-momentum limit of the self-energy when it is taken in an arbitrary direction close to the origin of the external momenta plane. The approach used here is the same developed in the first of the references in [20] to analyze the non-analyticity in the context of QFTFT. We are interested in the correction to the mass of the real $B(x)$ field when this is propagating along an arbitrary direction. We consider the analytic extension of expression (17) for small values of $z$ by taking into account the factors $\exp ( \pm i s z)$. This all means that, for small values of momenta, $z$ is a continuous variable and we can write the components of the external momentum $k_{\mu}$ in polar coordinates, as follows

$$
k_{\perp}=k \cos \varphi, k_{3}=k \sin \varphi \quad \text { with }-(\pi / 2) \leq \varphi \leq(\pi / 2),
$$

in such a way that expression (17) becomes

$$
\begin{array}{r}
I(z, v, u, s, \theta)=I(\xi, \varphi, u, s, \theta)=\frac{1}{\xi \cos \varphi}\left[\ln \left(\frac{\xi+2\left(u \cos \varphi+i \sqrt{1+u^{2}} \sin \varphi\right)}{\xi-2\left(u \cos \varphi-i \sqrt{1+u^{2}} \sin \varphi\right)}\right)\right. \\
\left.\times\left(n_{B}\left(s \sqrt{1+u^{2}}+2 \pi i \theta\right)+n_{B}\left(s \sqrt{1+u^{2}}-2 \pi i \theta-i s \xi \sin \varphi\right)\right)\right]+ \text { C.C. },
\end{array}
$$

where we have defined the dimensionless quantity $\xi=k / m$.

The zero-momentum limit $(k \rightarrow 0)$ is taken by maintaining $\varphi$, such that we can control the direction we approach the origin of the momentum plane. In this way we have

$$
\begin{array}{r}
\lim _{\xi \rightarrow 0} I(\xi, \varphi, u, s, \theta)=\frac{2 u}{u^{2}+\sin ^{2} \varphi} n_{B}\left(s \sqrt{1+u^{2}}+2 \pi i \theta\right)+ \\
+\left[i \ln \left(\frac{+2 u \cos \varphi+2 i \sqrt{1+u^{2}} \sin \varphi}{-2 u \cos \varphi 2 i \sqrt{1+u^{2}} \sin \varphi}\right) \tan \varphi s \exp \left(s \sqrt{1+u^{2}}+2 \pi i \theta\right)\left(n_{B}\left(s \sqrt{1+u^{2}}+2 \pi i \theta\right)\right)^{2}\right]+C . C . .
\end{array}
$$

Note that for $\varphi=0$, which corresponds to $k_{3}=0$ and a propagation perpendicular to the $x_{3}$ axis, expressions (31) and (19) are identical to each other, as they might be. Similarly, for a propagation parallel to the $x_{3}$ axis we take $\varphi= \pm \pi / 2$ and the expression (31) becomes equivalent to (25). This last case can be obtained by using the fact that

$$
\lim _{\varphi \rightarrow \pm \pi / 2}\left[\tan \varphi \ln \left(\frac{2 u \cos \varphi+2 i \sqrt{1+u^{2}} \sin \varphi}{-2 u \cos \varphi+2 i \sqrt{1+u^{2}} \sin \varphi}\right)\right]=-2 i \frac{u}{\sqrt{1+u^{2}}} .
$$


We would like to point out that the logarithm that appears in equation (31) is pure imaginary, namely

$$
\ln \left(\frac{+2 u \cos \varphi+2 i \sqrt{1+u^{2}} \sin \varphi}{\left.-2 u \cos \varphi+2 i \sqrt{1+u^{2}} \sin \varphi\right)}\right)=i\left[2 \arctan \left(\frac{\sqrt{1+u^{2}}}{u} \tan \varphi\right)-\pi \operatorname{sgn}\left(\frac{\sqrt{1+u^{2}}}{u} \tan \varphi\right)\right],
$$

where

$$
\operatorname{sgn}(x)=\left\{\begin{array}{ccc}
1 & \text { if } & x>0 \\
0 & \text { if } & x=0 \\
-1 & \text { if } & x<0
\end{array} .\right.
$$

By substituting (31) into (16) and using the definition of $s(15)$, we have that the correction to the mass when the $B(x)$ field propagates along an arbitrary direction in fact depends on the direction we approach the origin of the external momenta plane since the result depends on $\varphi$, namely

$$
\begin{array}{r}
\tilde{\Pi}\left(a, \theta, m, \mathbf{k}_{\perp}=0, k_{3}=0\right)=g^{2} \frac{1}{(2 \pi)^{2}} \frac{1}{4} \int_{0}^{\infty} d u \frac{u}{\sqrt{1+u^{2}}}\left[\frac{2 u}{u^{2}+\sin ^{2} \varphi} n_{B}\left(m a \sqrt{1+u^{2}}+2 \pi i \theta\right)+\right. \\
\left.+\left(i \ln \left(\frac{+2 u \cos \varphi+2 i \sqrt{1+u^{2}} \sin \varphi}{-2 u \cos \varphi+2 i \sqrt{1+u^{2}} \sin \varphi}\right)(\tan \varphi) m a \exp \left(m a \sqrt{1+u^{2}}+2 \pi i \theta\right)\left(n_{B}\left(m a \sqrt{1+u^{2}}+2 \pi i \theta\right)\right)^{2}\right)\right] .
\end{array}
$$

For $\theta=0$ and $\theta=1 / 2$ we have the cases of periodic and anti-periodic boundary conditions, respectively. We could take $\theta=0$ or $\theta=1 / 2$ from the beginning of the calculations, such that the results would be the same as the ones obtained from (35).

\section{CONCLUSIONS AND FINAL REMARKS}

In this paper we have found the correction to the mass for a real scalar field, in $3+1$ dimensions and in the static limit, due to quantum fluctuations of a complex scalar field with one spatial dimension compactified. Specifically, we have submitted the real scalar field to periodic conditions and the complex scalar field to quasi-periodic conditions. The results interpolate continuously the periodic and anti-periodic cases for the complex field.

We have shown that, despite the fact that the correction to the mass exhibits a dependence on the direction we approach the origin of the external momenta plane, the results for propagations parallel to and perpendicular to the compactified axis are equal to each other, that is, we have the same contribution to the mass for the cases where $\varphi=0$ and $\varphi= \pm \pi / 2$. As can be seen from (35), this curious fact does not happen for any other propagation direction.
It would be interesting to extend these results to the same model but in $4+1$ dimensions, in order to investigate if it is possible to have a mechanism of mass generation for fields due to the existence of an extra spatial dimensions compactified.

\section{Appendix}

The generating functional for the Lagrangian (1) is given by the path integral

$$
Z[J]=\int \mathcal{D} \phi_{\theta} \int \mathcal{D} B_{P} \exp \left[-\int d^{4} x[\mathcal{L}+J(x) B(x)]\right]
$$

where $\mathcal{D} \phi_{\theta}$ and $\mathcal{D} B_{P}$ means that the integrals must be taken only over field configurations that satisfies the conditions (2) and (3).

By following the same procedure used in quantum field theory without boundary conditions, we write

$$
\phi=\frac{1}{\sqrt{2}}\left(\phi_{1}+i \phi_{2}\right)
$$

what allows us to integrate (36) on the fields $\phi_{1}$ and $\phi_{2}$

$$
Z[J]=\int \mathcal{D} B_{P} \exp [-\operatorname{tr} \ln (1-g \hat{G} B)] \exp \left[-\int d^{4} x \frac{1}{2}\left(\left(\partial_{\mu} B\right)\left(\partial_{\mu} B\right)+M^{2} B^{2}\right)+J B\right],
$$

where we have discarded a normalization factor, $\hat{G}$ is the propagator operator of the scalar field $\phi_{\theta}(x)$ under the boundary condition (3) and we have used the definition (1).

Up to second order in the coupling constant $g$, we can write expression (38) in the form

$$
Z[J]=\int \mathcal{D} B_{P} \exp \left[-\int d^{4} x \frac{1}{2}\left(\left(\partial_{\mu} B\right)\left(\partial_{\mu} B\right)+M^{2} B^{2}\right)+(J-g G(0)) B\right]
$$




$$
\exp \left[-\frac{g^{2}}{2} \iint d^{4} x d^{4} y B(y) G(y, x) G(x, y) B(x)\right]
$$

where $G(x, y)=\langle x|\hat{G}| y\rangle$ is the Green's function of the scalar field with the conditions (3) and $G(0)=G(x, x)$. From the above expression one can define a new external current $J^{\prime}=J-g G(0)$ and identify the effective action for the $B$ field

$$
S_{e f f}=\int d^{4} x \frac{1}{2}\left(\left(\partial_{\mu} B\right)\left(\partial_{\mu} B\right)+M^{2} B^{2}\right)+\iint d^{4} x d^{4} y\left(\frac{g^{2}}{2} B(y) G(y, x) G(x, y) B(x)\right) .
$$

It is convenient to write the $S_{\text {eff }}$ in momentum space. For this task we consider that, due to the conditions (2), the $B$ field can be written in the form

$$
B(y)=\int \frac{d^{3} k_{\perp}}{(2 \pi)^{3}} \sum_{n=-\infty}^{\infty} \tilde{B}_{n}\left(k_{\perp}\right) e^{i k_{\perp} \cdot y_{\perp}} \psi_{n}\left(y^{3}\right),
$$

where quantities with subscript $\perp$ are to be read as those transverse to the compactified axis and

$$
\psi_{n}\left(y^{3}\right)=\frac{1}{\sqrt{a}} \exp \left(\frac{2 n \pi i}{a}\right) .
$$

Due to (3), the Green's function $G(x, y)$ is written as

$$
G(x, y)=\int \frac{d^{3} k_{\perp}}{(2 \pi)^{3}} e^{i p_{\perp} \cdot\left(x_{\perp}-y_{\perp}\right)} \sum_{\ell=-\infty}^{\infty} \frac{\phi_{\ell}\left(x^{3}\right) \phi_{\ell}^{*}\left(y^{3}\right)}{p_{\perp}^{2}+q_{\ell}^{2}+m^{2}}
$$

where

$$
\phi_{\ell}\left(x^{3}\right)=\frac{1}{\sqrt{a}} \exp \left(i \frac{2 \ell \pi+\theta}{a}\right)
$$

and

$$
q_{\ell}=\frac{2 \ell \pi+\theta}{a}
$$

By substituting (41), (42), (43), (44) and (45) in (40) and using the identities

$$
\int d y_{\perp} e^{-i\left(k_{\perp}+p_{\perp}-p_{\perp}^{\prime}\right) \cdot y_{\perp}}=(2 \pi)^{3} \delta^{3}\left(k_{\perp}+p_{\perp}-p_{\perp}^{\prime}\right)
$$

and

$$
\int d y^{3} \psi_{n}\left(y^{3}\right) \phi_{\ell^{\prime}}^{*}\left(y^{3}\right) \phi_{\ell}\left(y^{3}\right)=\frac{1}{\sqrt{a}} \delta_{n+\ell-\ell^{\prime}, 0},
$$

where $\delta_{n+\ell-\ell^{\prime}, 0}$ is the Kronecker delta, we can rewrite the effective action (40) in the form (6) with the corresponding effective Lagrangian (4).

\section{Acknowledgments}

$\mathrm{MH}$ thanks to Conselho Nacional de Desenvolvimento Científico e Tecnológico (CNPq-Brasil) for the financial support. FAB thanks to Fundação de Amparo à Pesquisa do Estado de São Paulo (FAPESP) for the financial support.
[1] T. Kaluza, Akad. Wiss. Phys. Math. K 1, 966 (1921).

[2] O. Klein, Z. Phys. 37, 895 (1926).

[3] Thomas Appelquist, Bogdan A. Dobrescu, Eduardo Ponton, and Ho-Ung Yee, Phys. Rev. Lett. 87, 181802 (2001) [arXiv:hep-ph/0107056v2].

[4] Lawrence Hall, Yasunori Nomura, Takemichi Okui, and David Smith, Phys. Rev. D 65, 035008 (2002) [arXiv:hep$\mathrm{ph} / 0108071 \mathrm{v} 4]$.

[5] Csaba Csaki, Christophe Grojean, and Hitoshi Murayama, Phys. Rev. D 67, 085012 (2003) [arXiv:hep-ph/0210133v2].

[6] Hsin-Chia Cheng, Bogdan A. Dobrescu, and Christopher T. Hill, Nucl. Phys. B 589, 249 (2000) [arXiv:hep-ph/9912343v3].

[7] Nima Arkani-Hamed, Hsin-Chia Cheng, Bogdan A. Dobrescu, and Lawrence J. Hall, Phys. Rev. D 62, 096006 (2000) [arXiv:hep-ph/0006238v2]; Michio Hashimoto, Masaharu Tanabashi and Koichi Yamawaki, Phys. Rev. D64, 056003 (2001) [arXiv:hep-ph/0010260v5].

[8] David J. Toms, J. Phys. A: Math. Gen. 36, 5121 (2003); S. J.
Avis and C. J. Isham, Proc. R. Soc. A 363, 581 (1978).

[9] Eduardo Ponton and Erich Poppitz, JHEP 0106, 019 (2001) [arXiv:hep-ph/0105021v3].

[10] R. Amorim and J. Barcelos-Neto, Braz. Jour. Phys. 32, 227 (2002) [arXiv:hep-th/0108171v2].

[11] L. M. Abreu, C. de Calan, A. P. C. Malbouisson, J. M. C. Malbouisson, and A. E. Santana, Jour. Math. Phys. 46, 012304 (2005); F.C. Khanna, A.P.C. Malbouisson, J.M.C. Malbouisson, H. Queiroz, T.M. Rocha-Filho, A.E. Santana and J.C. da Silva, Phys. Lett. B 624, 316 (2005).

[12] Bogdan A. Dobrescu and Eduardo Pontón, JHEP 03, 071 (2004).

[13] S. A. Fulling, Phys. Rev. D 7, 2850 (1973).

[14] D. J. Toms, Phys. Rev. D 21, 928 (1980).

[15] G. Denardo and E. Spallucci, Nucl. Phys. B 169, 514 (1980).

[16] L. H. Ford and T. Yoshimura, Phys. Lett. A 70, 89 (1979).

[17] L. H. Ford, Phys. Rev. D 21, 933 and 949 (1980).

[18] A. Das and M. Hott, Mod. Phys. Lett. A 10, 893 (1995). 
[19] L. Ford and N. F. Svaiter, Phys. Rev. D 51, 6981 (1995).

[20] H. Arthur Weldon, Phys. Rev. D 47, 594 (1993); ibid. 65, 076010 (2002).

[21] A. Das, Finite-Temperature Field Theory (World Scientific, New York, 1997).

[22] P. Arnold, S. Vokos, P. F. Bedaque, and A. Das, Phys. Rev. D 47, 4698 (1993)

[23] A. Das and M. Hott, Phys. Rev. D 50, 6655 (1994).

[24] R. J. Rivers, Path integral methods in Quantum Field Theory
(Cambridge University Press, Cambridge, 1987).

[25] H. Boschi-Filho, C. Farina, and A. de Souza Dutra, J. Phys. A 28, L7 (1995); H. Boschi-Filho, C. Farina, Phys. Lett. A 205, 255 (1995).

[26] P. F. Borges, H. Boschi-Filho, and C. Farina, Mod. Phys. Lett. A 13, 843 (1998); ibid. 14, 1217 (1999).

[27] The time-component of the momentum is part of $\mathbf{k}_{\perp}$, that is, $\mathbf{k}_{\perp}=\left(k_{0}, k_{1}, k_{2}\right)$ 